Acta Crystallographica Section D

Biological

Crystallography

ISSN 1399-0047

\title{
Structural evidence for the partially oxidized dipyrromethene and dipyrromethanone forms of the cofactor of porphobilinogen deaminase: structures of the Bacillus megaterium enzyme at near-atomic resolution
}

\author{
N. Azim, ${ }^{a}$ E. Deery, ${ }^{\text {b }}$ M. J. \\ Warren, ${ }^{b}$ B. A. A. Wolfenden, ${ }^{c}$ \\ P. Erskine, ${ }^{c}$ J. B. Cooper, ${ }^{{ }^{*}}$ * \\ A. Coker, ${ }^{\text {C }}$ S. P. Wood ${ }^{\mathrm{C}}$ and \\ M. Akhtar ${ }^{\mathrm{a}}$
}

aschool of Biological Sciences, University of Punjab, New Campus, Lahore-54590, Pakistan, ${ }^{\mathbf{b}}$ School of Biosciences, University of Kent, Stacey Building, Canterbury CT2 7NJ, England, and 'Laboratory of Protein Crystallography, Centre for Amyloidosis and Acute Phase Proteins, UCL Division of Medicine (Royal Free Campus), Rowland Hill Street, London NW3 2PF, England

Correspondence e-mail: jon.cooper@ucl.ac.uk
The enzyme porphobilinogen deaminase (PBGD; hydroxymethylbilane synthase; EC 2.5.1.61) catalyses an early step of the tetrapyrrole-biosynthesis pathway in which four molecules of the monopyrrole porphobilinogen are condensed to form a linear tetrapyrrole. The enzyme possesses a dipyrromethane cofactor, which is covalently linked by a thioether bridge to an invariant cysteine residue (Cys241 in the Bacillus megaterium enzyme). The cofactor is extended during the reaction by the sequential addition of the four substrate molecules, which are released as a linear tetrapyrrole product. Expression in Escherichia coli of a His-tagged form of B. megaterium PBGD has permitted the X-ray analysis of the enzyme from this species at high resolution, showing that the cofactor becomes progressively oxidized to the dipyrromethene and dipyrromethanone forms. In previously solved PBGD structures, the oxidized cofactor is in the dipyromethenone form, in which both pyrrole rings are approximately coplanar. In contrast, the oxidized cofactor in the $B$. megaterium enzyme appears to be in the dipyrromethanone form, in which the $\mathrm{C}$ atom at the bridging $\alpha$-position of the outer pyrrole ring is very clearly in a tetrahedral configuration. It is suggested that the pink colour of the freshly purified protein is owing to the presence of the dipyrromethene form of the cofactor which, in the structure reported here, adopts the same conformation as the fully reduced dipyrromethane form.

\section{Introduction}

Tetrapyrroles such as haem and chlorophyll play vital physiological roles in respiration and photosynthesis (Warren \& Smith, 2009). One of the early steps in the biosynthesis of tetrapyrroles is catalysed by the enzyme porphobilinogen deaminase (PBGD), which is also known as hydroxymethylbilane synthase (EC 2.5.1.61). This enzyme catalyses the polymerization of four molecules of the monopyrrole porphobilinogen in a stepwise head-to-tail manner to form the linear tetrapyrrole preuroporphyrinogen or hydroxymethylbilane (Fig. 1; Jordan, 1991). PBGDs are monomeric enzymes with molecular masses in the range $34-44 \mathrm{kDa}$ depending on the species. The enzymes in this family exhibit high thermal stabilities and have $\mathrm{pH}$ optima in the range 8.0-8.5, with isoelectric points between 4.0 and 4.5 . It has been shown that ring $A$ of the tetrapyrrole product (Fig. 1) is the first to bind to the enzyme, followed by rings $B, C$ and finally $D$ (Jordan, 1991). The enzyme possesses a dipyrromethane cofactor (Fig. 2) that is covalently bound to the enzyme by a thioether linkage involving an invariant cysteine residue (Cys241 in the Bacillus megaterium enzyme; Jordan \& Warren, 1987; Warren
Received 10 September 2013 Accepted 4 December 2013

PDB references: porphobilinogen deaminase, $4 \mathrm{mlv} ; 4 \mathrm{mlq}$ 
\& Jordan, 1988; Scott et al., 1988). Whilst the cofactor can be assembled slowly from two molecules of porphobilinogen, it can be generated more quickly by cleavage of the product, preuroporphyrinogen, which reacts rapidly with the apoenzyme (Scott et al., 1989; Awan et al., 1997). During catalysis, the free $\alpha$-position of the cofactor acts as the attachment point to which the growing tetrapyrrole chain is anchored. The four porphobilinogen substrate molecules (S) react sequentially with the enzyme (E) to generate stable $\mathrm{ES}_{1}, \mathrm{ES}_{2}, \mathrm{ES}_{3}$ and $\mathrm{ES}_{4}$ complexes. $\mathrm{ES}_{4}$ is therefore an enzyme-bound hexapyrrole, representing two pyrroles of the cofactor attached to the tetrapyrrole bilin product. After the assembly of $\mathrm{ES}_{4}$, cleavage of the link between the cofactor and the first substrate molecule completes the reaction, allowing the tetrapyrrole product to be released.

The X-ray structures of the Escherichia coli, human and Arabidopsis enzymes have been solved (Louie et al., 1992, 1996; Hädener et al., 1999; Gill et al., 2009; Song et al., 2009; Roberts et al., 2013). The polypeptide is folded into three domains (1-3), each of approximately the same size. The topology of domains 1 and 2 shows a strong resemblance to the type II periplasmic binding proteins (Louie et al., 1992; Louie, 1993), whereas domain 3 has an entirely distinct fold. The dipyrromethane cofactor is covalently attached to a cysteine residue in a loop of domain 3 so that it is positioned within a deep active-site cleft formed between domains 1 and 2 . The active-site cleft is formed by several crucial arginine residues that bind the side-chain carboxylates of the cofactor and/or substrate. Indeed, many of the point mutations in the human PBGD gene which give rise to the disease acute intermittent porphyria affect these conserved arginine residues (Wood et al., 1995).

B. megaterium, literally meaning 'big beast' (Vary, 1994), is a common Gram-positive soil bacterium that is highly versatile as its metabolism allows it to utilize inexpensive carbon sources and is capable of surviving in a divergent range of environments (Vary et al., 2007). It has many commercial

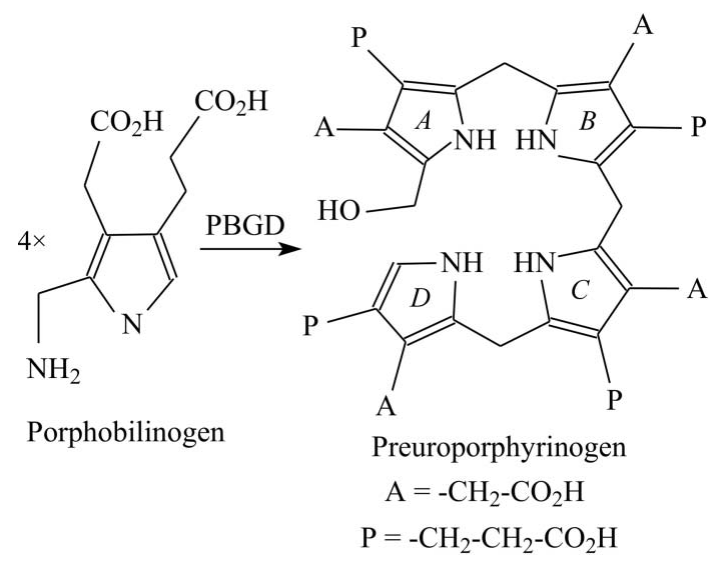

Figure 1

The reaction catalysed by porphobilinogen deaminase. Four molecules of the pyrrole porphobilinogen are condensed to form the linear tetrapyrrole preuroporphyrinogen (hydroxymethylbilane). The acetic acid and propionic acid side chains of each pyrrole are abbreviated $\mathrm{A}$ and $\mathrm{P}$, respectively, and the four rings of the tetrapyrrole product are indicated in italics as $A, B, C$ and $D$. applications in food processing and in the biotechnological production of various drugs and vitamins, including the tetrapyrrole-derived vitamin $\mathrm{B}_{12}$ (Bunk et al., 2010). The gene for PBGD in B. megaterium encodes a protein of 310 amino acids which has $48 \%$ sequence identity to the $E$. coli enzyme. Here, we report the structure analysis of PBGD from $B$. megaterium in a crystal form that diffracts synchrotron radiation to $1.5 \AA$ resolution. The collection of data from two crystals, which were flash-cooled at periods of 40 and $50 \mathrm{~d}$ after purification, demonstrates that the dipyrromethane cofactor adopts two conformations corresponding to partially oxidized states. One of these is likely to be the dipyrromethene form, as it adopts essentially the same conformation as the fully reduced dipyrromethane. The other is likely to be the further oxidized dipyrromethanone form, which has not previously been observed structurally. This form possesses a tetrahedral ring-linking $\alpha$-C atom in the terminal pyrrole, and the two structures presented here demonstrate that the proportion of the cofactor in this state increases as a function of time, with a concomitant decrease in the amount of the other conformer.

\section{Methods}

The expression of B. megaterium PBGD in E. coli using a pET-14b construct has been reported recently along with the purification and crystallization of the enzyme (Azim et al., 2013). The affinity-purified enzyme was assayed spectrophotometrically using the method described in ShoolinginJordan et al. (1997). Since the dipyrromethane cofactor of PBGD is light-sensitive, crystals were grown in the dark using the hanging-drop method. Crystals of the enzyme could be obtained reproducibly in $0.1 \mathrm{M}$ sodium cacodylate $\mathrm{pH}$ 6.5-6.8, $0.2 M$ magnesium acetate, $25-30 \%$ PEG $8 \mathrm{~K}$ following removal of the polyhistidine tag and concentration of the enzyme to $2.5 \mathrm{mg} \mathrm{ml}^{-1}$. Selected crystals were treated by the addition of glycerol to approximately $30 \%(v / v)$ before mounting in loops and flash-cooling with an Oxford Cryosystems cryocooler. Data were collected from two crystals, one of which (crystal 1) was cooled approximately $40 \mathrm{~d}$ after purification of the protein and the other of which (crystal 2) was cooled when the protein was approximately $50 \mathrm{~d}$ old.

X-ray data collection on crystal 1 was undertaken at station I03 at the Diamond Light Source (DLS; Didcot, England) using a Pilatus 6M-F detector at a wavelength of $0.976 \AA$ A. Data

\section{Figure 2}

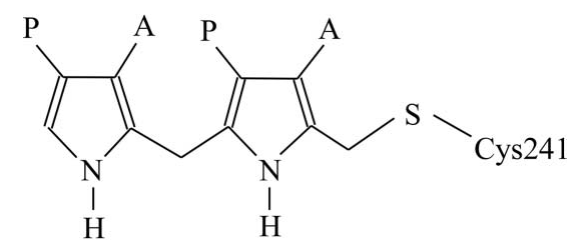

The dipyrromethane cofactor of porphobilinogen deaminase is covalently attached to the enzyme by a thioether bond to a cysteine residue. Four substrate pyrroles are added linearly to the cofactor; finally, hydrolysis of the linkage between the substrate and the cofactor releases the tetrapyrrole product hydroxymethylbilane. 
Table 1

Data-collection and processing statistics for B. megaterium porphobilinogen deaminase.

Values in parentheses are for the outer resolution shell.

\begin{tabular}{|c|c|c|}
\hline Data set & Crystal 1 & Crystal 2 \\
\hline Beamline & I03, DLS & BM14, ESRF \\
\hline Wavelength (̊̊) & 0.976 & 1.072 \\
\hline Space group & $P 2_{1} 2_{1} 2_{1}$ & $P 2_{1} 2_{1} 2_{1}$ \\
\hline \multicolumn{3}{|l|}{ Unit-cell parameters $(\AA)$} \\
\hline$a$ & 53.32 & 53.01 \\
\hline$b$ & 65.78 & 65.12 \\
\hline$c$ & 97.21 & 96.78 \\
\hline Mosaic spread $\left({ }^{\circ}\right)$ & 0.26 & 1.01 \\
\hline Resolution (A) & $\begin{array}{l}48.60-1.46 \\
\quad(1.53-1.46)\end{array}$ & $\begin{array}{l}31.33-1.60 \\
\quad(1.69-1.60)\end{array}$ \\
\hline$R_{\text {merge }} \dagger(\%)$ & $6.1(55.9)$ & $8.8(149.3)$ \\
\hline $\mathrm{CC}_{1 / 2} \neq(\%)$ & $99.8(86.7)$ & $99.8(33.1)$ \\
\hline$R_{\text {meas }} \S(\%)$ & $6.7(61.3)$ & $9.5(168.4)$ \\
\hline Completeness (\%) & $100.0(100.0)$ & $95.6(88.7)$ \\
\hline Average $I / \sigma(I)$ & $14.4(3.0)$ & $10.1(0.9)$ \\
\hline Multiplicity & $6.2(6.0)$ & $6.4(4.5)$ \\
\hline No. of observed reflections & $378329(52575)$ & $273058(25347)$ \\
\hline No. of unique reflections & $60772(8748)$ & $42866(5649)$ \\
\hline Wilson plot $B$ factor $\left(\AA^{2}\right)$ & 15.8 & 17.0 \\
\hline$R$ factor $(\%)$ & 14.1 & 16.1 \\
\hline Free $R$ factor (\%) & 18.6 & 24.2 \\
\hline R.m.s.d., bond lengths ( & 0.024 & 0.019 \\
\hline R.m.s.d., bond angles $\left(^{\circ}\right)$ & 2.44 & 2.14 \\
\hline No. of reflections in working set & 57626 & 40552 \\
\hline Mean protein $B$ factor $\left(\AA^{2}\right)$ & 24.6 & 28.2 \\
\hline
\end{tabular}

$\dagger R_{\text {merge }}=\sum_{h k l} \sum_{i}\left|I_{i}(h k l)-\langle I(h k l)\rangle\right| / \sum_{h k l} \sum_{i} I_{i}(h k l) . \quad \neq \mathrm{CC}_{1 / 2}$ is the half-set correlation coefficient as described by Karplus \& Diederichs (2012). $\S R_{\text {meas }}=\sum_{h k l}\{N(h k l) /$ $[N(h k l)-1]\}^{1 / 2} \sum_{i}\left|I_{i}(h k l)-\langle I(h k l)\rangle\right| / \sum_{h k l} \sum_{i} I_{i}(h k l)$, where $\langle I(h k l)\rangle$ is the mean intensity of the $N(h k l)$ observations $I_{i}(h k l)$ of each unique reflection $h k l$ after scaling.

were collected from crystal 2 on the European Synchrotron Radiation Facility (ESRF) beamline BM14 using a MAR Mosaic $225 \mathrm{CCD}$ detector at a wavelength of $1.07 \AA$. Both data sets were collected at a temperature of $100 \mathrm{~K}$ using $1^{\circ}$ oscillations and were processed with MOSFLM (Leslie, 2006; Powell et al., 2013), SCALA (Evans, 2006) and other programs from the $C C P 4$ suite (Winn et al., 2011). Structure analysis was successful for the crystal 1 data set using the molecularreplacement program MOLREP (Vagin \& Teplyakov, 2010) with E. coli PBGD (48\% identity; PDB entry 1pda; Louie et $a l ., 1992)$ as the search model. Refinement and rebuilding of the $B$. megaterium PBGD structure was completed using this data set with the programs REFMAC (Murshudov et al., 2011) and Coot (Emsley \& Cowtan, 2004). Subsequently, the structure was refined as above using the crystal 2 data set, and further crystallographic details for both structures can be found in Table 1. The geometric restraints for refinement of the two forms of the cofactor were generated using PRODRG (Schüttelkopf \& van Aalten, 2004). The final refined structures and reflection data sets were analysed by the validation programs PROCHECK (Laskowski et al., 1993), SFCHECK (Vaguine et al., 1999) and RAMPAGE (Lovell et al., 2003; Chen et al., 2010) and have been deposited in the Protein Data Bank (http://www.wwpdb.org) with accession codes $4 \mathrm{mlv}$ and $4 \mathrm{mlq}$ for crystals 1 and 2, respectively. Figures of the structures were prepared using CueMol (http://www.cuemol.org/en) and were rendered using $P O V$-Ray (http://www.povray.org).

\section{Results}

\subsection{Structure analysis}

The structure of $B$. megaterium PBGD has been determined at a resolution of $1.46 \AA$, with an $R$ factor of $14.1 \%$ and an $R_{\text {free }}$ of $18.6 \%$ (see Table 1 ). The resulting model of the enzyme was found to have $99 \%$ of the amino acids within the favoured regions of the Ramachandran plot and the remaining $1 \%$ within allowed areas according to the RAMPAGE criteria (Lovell et al., 2003). The estimated r.m.s. coordinate error (Read, 1986) of $0.11 \AA$ suggests that the structure is defined with high accuracy. In addition, the structure of the enzyme in which the cofactor has undergone more extensive oxidation is presented at the slightly lower resolution of $1.6 \AA$ with similar refinement and validation statistics (see Table 1). The overall fold of the enzyme is shown in Fig. 3, in which the secondarystructure elements are labelled according to the notation of Louie et al. (1992).

All enzymes in this family adopt a three-domain fold in which domains 1 and 2 resemble the fold of type II periplasmic binding proteins and the third domain, to which the cofactor is covalently attached, adopts a distinct $\alpha+\beta$ topology. Starting at the N-terminal end, the backbone of B. megaterium PBGD follows that of the $E$. coli enzyme until the end of stand $\beta 2_{1}$, where there is a substantial disordered region extending from residues 42 to 62 . This disordered region is known to form a flexible loop covering the active site and has only been defined structurally in the Arabidopsis enzyme (Roberts et al., 2013). The ordered region of the B. megaterium structure resumes at residue 63 , which is at the $\mathrm{N}$-terminal end of helix $\alpha 2_{1}$, and

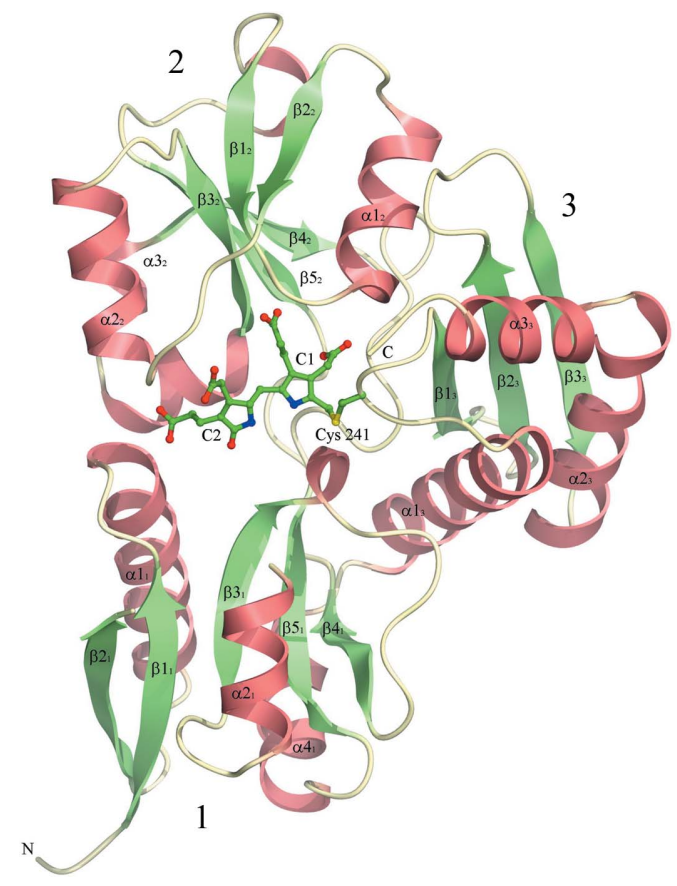

Figure 3

The tertiary structure of $B$. megaterium PBGD at $1.46 \AA$ resolution showing the oxidized form of the dipyrromethane cofactor covalently attached to Cys241. The domains of the enzyme are numbered 1-3 and the secondary-structure elements are labelled according to the nomenclature of Louie et al. (1992). 
continues to follow the fold of the E. coli enzyme closely, with the exception of a few single-residue insertions in loop regions that are distant from the active site. The sequence alignment shown in Fig. 4 shows the marked similarity of enzymes in this family. Not surprisingly, the eukaryotic enzymes exhibit some significant differences from the prokaryotic PBGDs, e.g. the human enzyme has a large insertion towards the C-terminal end and the Arabidopsis enzyme appears to be somewhat truncated relative to the others at the same end of the molecule.

The refined structure of $B$. megaterium PBGD superimposes with the E. coli and Arabidopsis enzymes (which are of comparable resolution) with r.m.s. $\mathrm{C}^{\alpha}$ deviations of 1.05 and $1.02 \AA$ for 280 and 271 structurally equivalent residues, respectively. Likewise, superposition with the structure of the human enzyme demonstrates a similarly good r.m.s.d. of $1.13 \AA$ for 276 structurally equivalent $\mathrm{C}^{\alpha}$ atoms. It has been suggested that the domains of this enzyme may move independently to allow substantial rearrangements of the polypyrrole during the elongation cycle (Louie et al., 1992, 1996). Inspection of the A. thaliana PBGD structure suggested that domains 2 and 3 of the enzyme appear to move as a relatively rigid unit with respect to domain 1 (Roberts et al., 2013). Indeed, the superpositions of B. megaterium PBGD with the E. coli, Arabidopsis and human enzymes which are shown in Fig. 5 corroborate this suggestion and demonstrate that the rigid body formed of domains 2 and 3 of the B. megaterium enzyme has a very similar orientation to that of the E. coli enzyme, with which it shares $48 \%$ sequence identity. Indeed, the domain shifts of the $B$. megaterium and E. coli enzymes seem to lie between the larger movements exhibited by the Arabidopsis and human PBGDs (Fig. 5), which have slightly lower sequence identities of 43 and $47 \%$ to the B. megaterium enzyme, respectively.

\subsection{Definition of the cofactor redox states}

In the electron-density maps for both structures presented here, the dipyrromethane cofactor, which is covalently attached to Cys241, is very well

Figure 4 defined (Fig. 6). The two rings of the cofactor (labelled $\mathrm{C} 1$ and C2) are held within a large highly conserved cleft between domains 1 and 2 of the protein. The cleft has a preponderance of basic residues, most notably arginine, which interact electrostatically with the carboxylate side groups of the pyrroles. At the base of the cleft, an invariant aspartic acid, Asp82 in B. megaterium PBGD, forms hydrogen bonds to both the $\mathrm{C} 1$ and $\mathrm{C} 2$ pyrrole-ring $\mathrm{N}$ atoms. These interactions have been described in great detail elsewhere for high-resolution structures of related enzymes (e.g. Louie et al., 1992, 1996; Roberts et al., 2013), and the high degree of conservation of these critical amino acids (see Fig. 4) means that essentially the same interactions are observed in the B. megaterium structure reported here. However, one interesting difference is that the flap covering the active site of $B$. megaterium PBGD (residues 42-62) completely lacks electron density, despite many efforts to rebuild it during refinement of the two structures reported
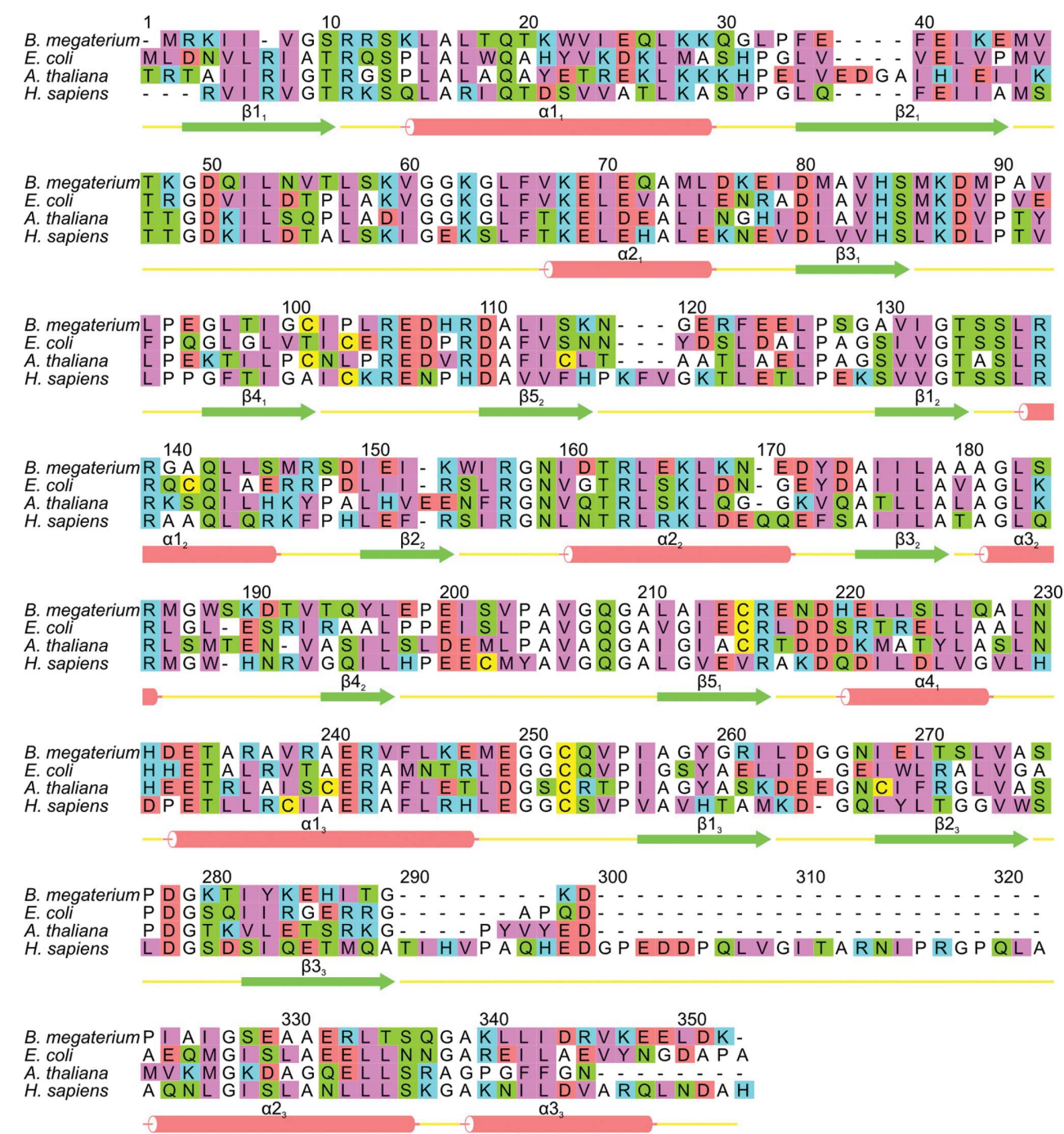

Sequence alignment and secondary structure of B. megaterium PBGD. An alignment of B. megaterium PBGD with the enzyme from another prokaryote $(E$. coli) along with the plant (A. thaliana) and human enzymes. The secondary-structure elements are labelled using the notation of Louie et al. (1992) and the amino-acid residues are colour-coded as follows: cyan, basic; red, acidic; green, neutral polar; pink, bulky hydrophobic; white, Gly, Ala and Pro; yellow, Cys. 
here. This suggests that the active-site flap of $B$. megaterium PBGD is rather more disordered than in the structures of other related enzymes. It is not immediately obvious why this should be the case since the flap sequence is quite highly conserved in PBGD enzymes (Fig. 4), although one factor may be the complete absence of crystal contacts in this region of

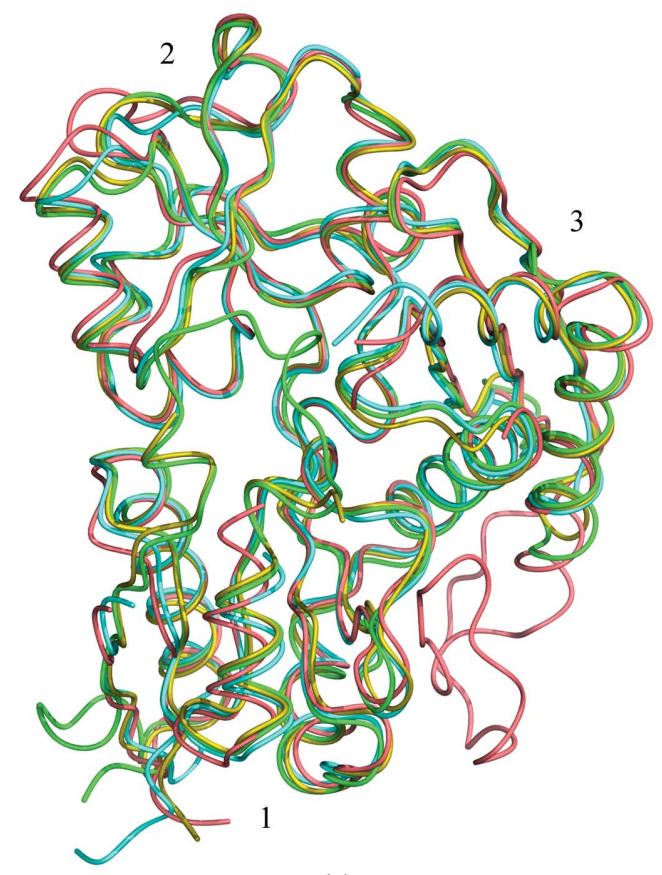

(a)

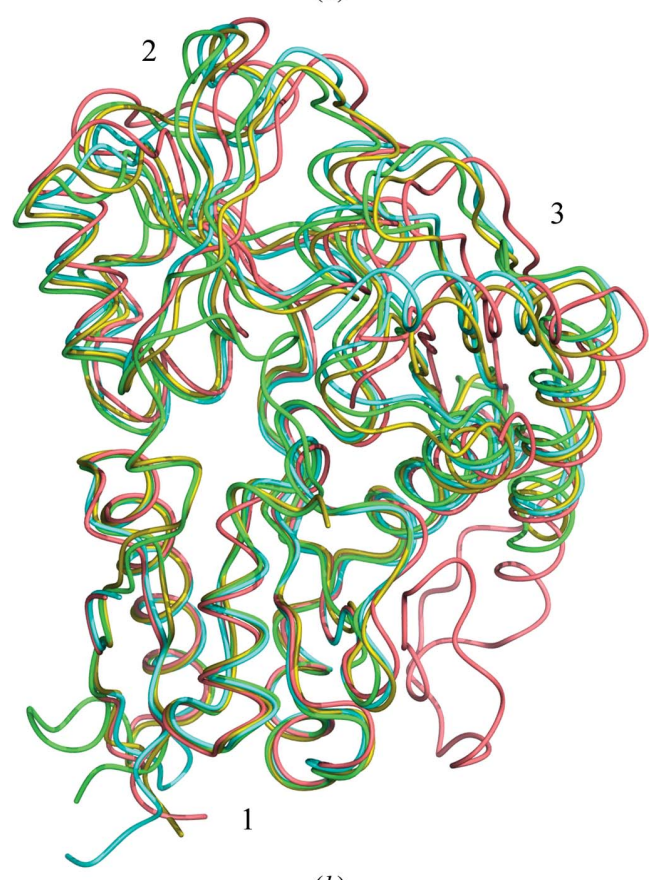

(b)

Figure 5

Superposition of B. megaterium PBGD with the Arabidopsis, E. coli and human enzymes. (a) The overall least-squares superposition of B. megaterium PBGD (cyan) with the E. coli enzyme (yellow) as well as the Arabidopsis and human enzymes (green and pink, respectively). A superposition of the four enzymes based on domain 1 alone is shown in (b), which emphasizes the different concerted shifts of domains 2 and 3 relative to domain 1 in the enzyme from each species. the $B$. megaterium structure. In principle, the greater flexibility of the flap may confer the cofactor of the B. megaterium enzyme with greater flexibility and sensitivity to environmental factors such as $\mathrm{pH}$, ionic strength and redox state. Indeed, reducing SDS-PAGE and mass spectrometry confirmed that cleavage of the enzyme occurs over a period of several days during storage on ice, giving a reduction in its molecular mass from 35 to $28 \mathrm{kDa}$. This is consistent with cleavage by trace proteases somewhere within the highly

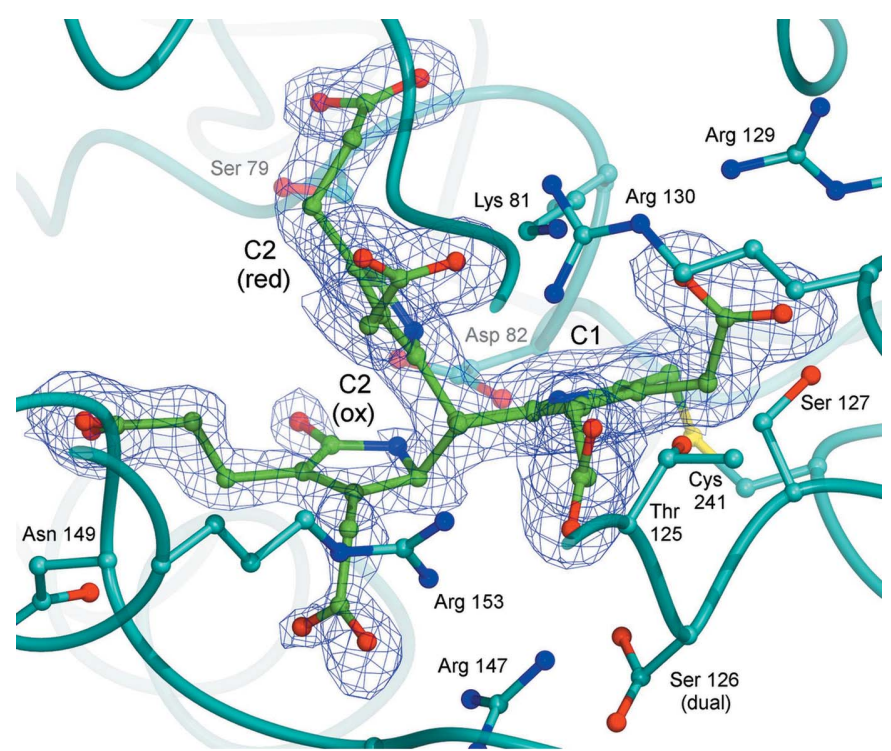

(a)

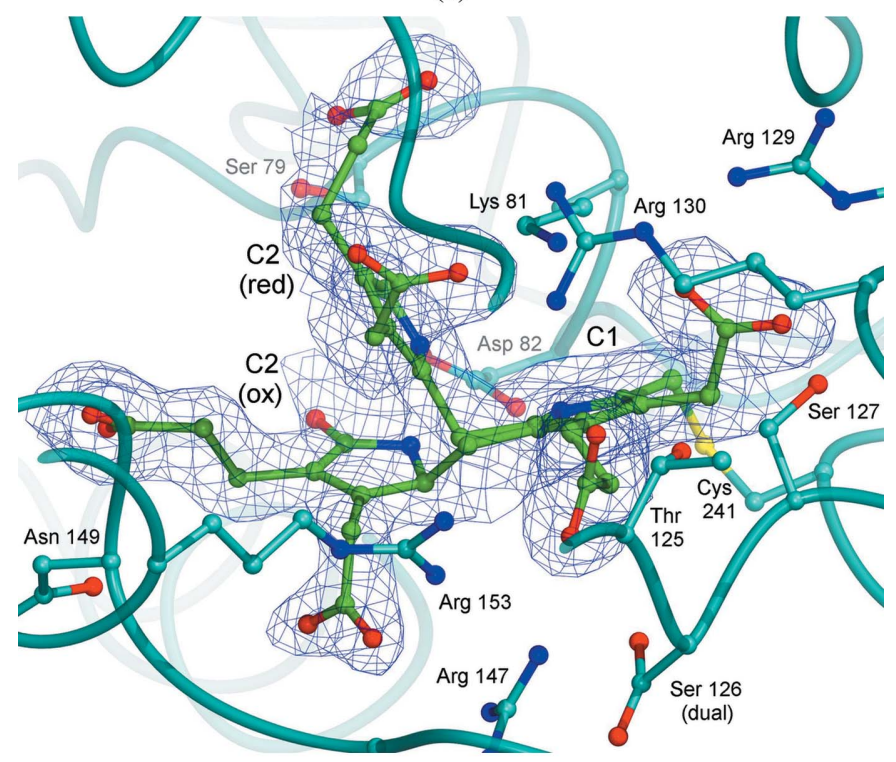

(b)

Figure 6

Electron density showing the dual conformations of the cofactor. The local fold of the protein is indicated as a cyan tube and the side chains adjacent to the cofactor are shown in the same colour as the enzyme, while the cofactor itself is coloured green. The map obtained for the 40-day-old protein is shown in $(a)$, with that for the 50-day-old protein shown in $(b)$; both maps are contoured at 0.7 r.m.s.. The $\mathrm{C} 2$ ring clearly adopts two positions depending on its oxidation state, which are shown as $\mathrm{C} 2$ (ox) and $\mathrm{C} 2$ (red). In the oxidized conformation, the $\mathrm{C} 2$ ring possesses a carbonyl $\mathrm{O}$ atom substituted at the $\alpha$-position; accordingly, the proportion of cofactor adopting this conformer increases with time. 
disordered active-site flap region between residues 42 and 62 during storage. It may be interesting that kinetic studies of the enzyme showed that whilst it has a $k_{\text {cat }}$ that is comparable to those of other PBGDs $\left(0.1 \mathrm{~s}^{-1}\right)$, its $K_{\mathrm{m}}$ of $100 \mu M$ is rather high for this enzyme family (Jordan, 1991), although it is comparable to the values found for algal PBGDs (Battersby et al., 1983). Hence, it is conceivable that a lower affinity for substrate may originate from greater disorder in the active-site flap region of the enzyme.

Another interesting feature of B. megaterium PBGD is the pink colouration which is found in the cells expressing the enzyme and in the final affinity-purified enzyme (Azim et al., 2013). Over time the protein solution becomes progressively yellow and the crystals we obtained were indeed of this colour, as is the case for most PBGDs studied to date. Prior to structure analysis of the B. megaterium enzyme, we anticipated that the initial pink coloration might indicate the presence of a previously unseen polypyrrole intermediate or an unusual oxidation product of the dipyrromethane cofactor. Structural studies of the E. coli and A. thaliana PBGD enzymes have shown that the cofactor becomes oxidized to the predominantly planar dipyrromethenone form, in which the $\alpha$-position of the terminal pyrrole ring (ring $\mathrm{C} 2$ ) is oxidized, as shown in Fig. 7. This form of the enzyme is catalytically inactive owing to the usual attachment site for incoming pyrroles being blocked by a carbonyl $\mathrm{O}$ atom: an effect that probably accounts for the slow inactivation of the enzyme over 2-3 weeks during storage or crystallization.

In both of the structures of B. megaterium PBGD that we report here, the $\mathrm{C} 2$ ring clearly adopts two positions (Fig. 6), one of which corresponds to the fully reduced dipyrromethane that has been observed in structures of the E. coli and human enzymes when they are crystallized under reducing conditions (Hädener et al., 1999; Song et al., 2009). The other position of the $\mathrm{C} 2$ ring corresponds approximately to that of the oxidized cofactor in the highresolution structures of the E. coli and Arabidopsis enzymes when the crystals were grown under oxidizing conditions (Louie et al., 1996; Roberts et al., 2013). In these structures, the $\mathrm{C} 2$ ring of the cofactor has a carbonyl oxygen substituent at the $\alpha$-posi-

Figure 7 tion (see Fig. 7a), which will render the enzyme inactive (see above). In the dipyrromethenone state, both rings of the cofactor are found to be approximately coplanar and, although they are not constrained to be so by the molecular geometry, this effect may facilitate the delocalization of electrons over both pyrrole rings. In the B. megaterium enzyme the electron-density map clearly shows that the oxidized conformation of the cofactor has the same oxygen substituent in the $\mathrm{C} 2$ ring, which is suggestive of the same dipyromethenone form. However, the other $\alpha$-position of the $\mathrm{C} 2$ ring which partakes in the methylene bridge with the adjacent $\mathrm{C} 1$ pyrrole is clearly tetrahedral, in contrast to the trigonal planar configuration that is found in dipyrromethenone. This suggests that in the B. megaterium PBGD structure the 'oxidized' conformation of the cofactor is actually the partly reduced dipyrromethanone form, the formula for which is shown in Fig. 7(a). Accordingly, refinement of the occupancies of the oxidized and reduced states in the two structures reported here showed that the proportion of the oxidized dipyrromethanone form increases in a time-dependent manner from
(1)<smiles>Pc1c[nH]c(Cc2[nH]c(CSC3CCCCC3)c([Tl])c2P)c1I</smiles>

(2)

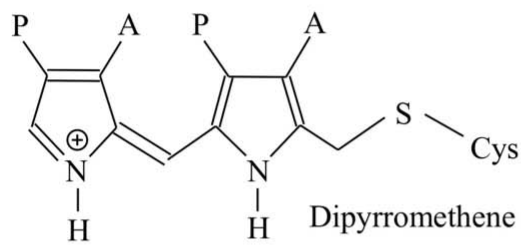

(3)<smiles>CC1=C(P)C(Cc2[nH]c(CSC3CCCCC3)c(P)c2I)NC1=O</smiles>

(4)

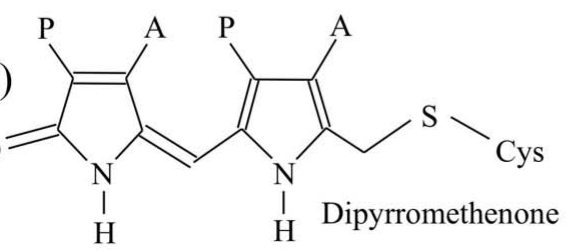

(a)
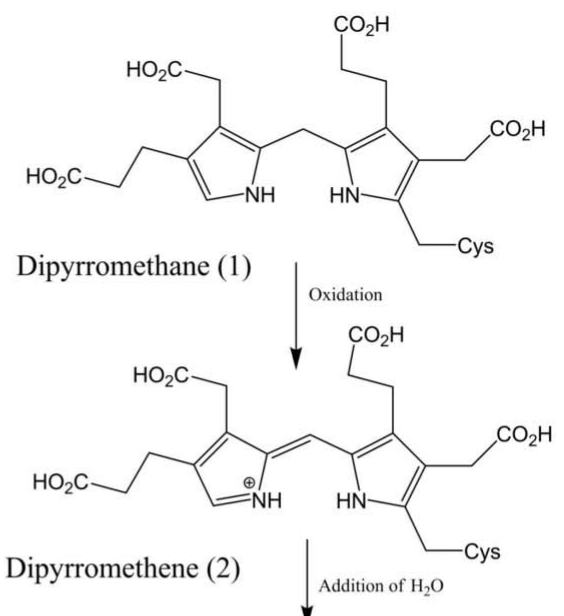<smiles>O=C(O)CCC1=C(CC(=O)O)C(O)(O)NC1=Cc1[nH]c(CO)c(CC(=O)O)c1CC(=O)O</smiles><smiles>CC1(C)NC(c2[nH]c(CO)c(CC(=O)O)c2CCC(=O)O)C(CCC(=O)O)=C1CC(=O)O</smiles>

Dipyrromethanone (3)

(b)

Oxidation states of the dipyrromethane cofactor. Different possible oxidation states of the dipyrromethane cofactor are shown in $(a)$ along with a proposed mechanism for oxidation of the dipyrromethane to dipyrromethene and subsequently dipyrromethanone $(b)$. 
$30 \%$ in the case of the 40 -day-old protein to $50 \%$ for the 50-day-old protein.

As mentioned above, the intriguing pink colouration of the freshly purified protein gradually changes to yellow over a 2-3-week period. One possibility is that the pink colour is owing to the dipyrromethene state (Fig. 7), which is derived from the fully reduced cofactor dipyrromethane by partial oxidation. Since the protonated dipyrromethene has a positive charge delocalized over two conjugated rings, one therefore might expect it to be more intensely coloured than the other intermediates shown in Fig. 7. In an electron-density map, a dipyrromethene would be expected to look exactly like the fully reduced dipyrromethane because it only differs from the latter by the loss of an $\mathrm{H}$ atom from the bridging $\mathrm{C}$ atom. The yellow colour that accumulates over time could therefore be owing to the dipyrromethanone (as shown in Fig. 7), which arises from further oxidation of the dipyrromethene. A proposed mechanism for this process is shown in Fig. 7(b).

To summarize, the native enzyme is most likely to contain a dipyrromethane, which oxidizes to a dipyrromethene with an extended chromophore giving the red-shifted spectrum. In a time-dependent fashion, the dipyrromethene is converted into the yellow dipyrromethanone form. The latter conversion, as shown in Fig. 7(b), merely requires the addition of water to the protonated Schiff base followed by prototropic rearrangements. According to this mechanism, the dipyrromethenone is the further oxidation product of the dipyrromethanone.

The two structures reported here superimpose very closely, with an r.m.s.d. of only $0.17 \AA$ for all $\mathrm{C}^{\alpha}$ atoms. Since analysis of the A. thaliana PBGD structure suggested that domains 2 and 3 appeared to move slightly relative to domain 1 of the enzyme as a rigid unit (Roberts et al., 2013), the same analysis was conducted for both of the B. megaterium structures. Interestingly, domains 2 and 3 appear to rotate towards domain 1 by approximately $0.6^{\circ}$ in the more oxidized structure, suggesting that as the proportion of cofactor in the more extended state increases, the active site of the enzyme is able to close more. This rigid-body rotation gives rise to movements of several loops over the active site of at most $0.5 \AA$. Whilst this domain movement would appear to be consistent with the more extended conformation of the oxidized cofactor, allowing the active-site cleft to close, it should be noted that the scale of the loop movements is of the order of the differences in unit-cell parameters. Thus, whether these domain movements are a cause or an effect of the slightly different crystal lattice parameters cannot easily be determined.

\section{Discussion}

Porphobilinogen deaminase is an intriguing enzyme and few details of how it catalyses the tetrapolymerization of pyrroles are well understood, although a number of possible models for the elongation process have been suggested (Jordan, 1991; Louie et al., 1992). One possibility is that domain movements cause the bound cofactor and polypyrrole intermediates to move past the catalytic centre of the enzyme in a stepwise manner, thus permitting the binding of additional substrate moieties and the completion of the tetrapyrrole product. This model would allow the condensation reactions to be driven by the extensive interactions that we observe between the enzyme and the cofactor, coupled with acid-base catalysis provided by the invariant aspartate residue (Asp82 in the PBGD from B. megaterium). Whilst the reported structure confirms all of these features, it is unusual in that the enzyme has a highly disordered flap over the active site, with some 20 amino-acid residues being invisible in the electron density. One consequence of this is that the full extent of the cavities around the active-site crevice becomes apparent and hints at the numerous possibilities for accommodating the polypyrrole intermediates of the elongation reaction. Another model of the elongation reaction is that the cofactor remains close to the positions observed in the X-ray structure as an incoming pyrrole is condensed with it. If the newly added pyrrole can then be repositioned, this local movement may be sufficient to 'free-up' the catalytic apparatus for the next elongation cycle. In the reduced conformation, the $\mathrm{C} 2$ ring of the cofactor is buried further within the active-site cavity, which creates more space for an incoming pyrrole to bind in the vicinity of the catalytic aspartate without displacement of the cofactor. However, the tight geometric constraints of the active site are such that steric hindrance between the cofactor and an incoming pyrrole would probably force the $\mathrm{C} 2$ ring into a conformation in which its free $\alpha$-position would be even less accessible to the incoming substrate. Regardless of the mechanism of elongation, movement of the cofactor is most likely to be linked to movement of the protein domains, and the current structure provides further corroboration for a model in which domains 2 and 3 of the protein appear to move relative to domain 1 as a fairly rigid unit (Roberts et al., 2013). In this respect, it is interesting that domains 2 and 3 provide the bulk of the binding interactions with the cofactor, whilst domain 1 provides the main catalytic residue of the enzyme, Asp82.

PBGD enzymes are highly sensitive to oxidation of the dipyrromethane cofactor, which causes the protein to become notably yellow with time (Jordan, 1991; Louie et al., 1996). Intriguingly, the B. megaterium enzyme has a pronounced pink colour during overexpression and purification (Azim et al., 2103), although the purified protein slowly becomes yellow over a period of a few weeks. Our structural analyses of the $B$. megaterium enzyme at two different time points after purification establish the different oxidation states of the cofactor that are likely to be responsible for these effects. The native enzyme will only be active with fully reduced dipyrromethane at the active site. However, the more exposed nature of the active-site cleft in this enzyme when compared with other PBGD structures suggests that the cofactor may be particularly prone to oxidation to the dipyrromethene form, which is highly conjugated and is likely to have a red-shifted spectrum when protonated. Protonation of the dipyrromethene gives it a positive charge on one of the pyrrole $\mathrm{N}$ atoms which can readily be stabilized by its close proximity to the catalytic aspartate Asp82. Whilst discrimination between 
dipyrromethene and dipyrromethane would not be possible by X-ray diffraction analysis at the resolutions reported here, there are compelling reasons to believe that the pink colouration is owing to the presence of the partly oxidized protonated dipyrromethene form. Addition of water to this dipyrrin converts it to the yellow dipyrromethanone form, which possesses a tetrahedral $\alpha$-C atom in the terminal pyrrole. The time-dependent accumulation of this intermediate is confirmed by our X-ray analyses. Further oxidation of the dipyrromethanone will ultimately lead to the dipyrromethenone form, which has been confirmed in other highresolution PBGD structures (Louie et al., 1996; Roberts et al., 2013). The loss of catalytic activity as the cofactor is oxidized to the dipyrromethanone form cannot be reversed by the addition of mild reducing agents. Indeed, there is no known reducing agent that will reverse the oxidized cofactor to its original redox state in a predictable fashion and still maintain the integrity of the protein structure.

We gratefully acknowledge the Pakistan Higher Education Commission for a scholarship to NA. We acknowledge Diamond Light Source (DLS; England) and the European Synchrotron Radiation Facility (ESRF; Grenoble, France) for beam time and travel support (award Nos. MX-7131 and MX-1425). We thank Drs Graham Taylor and Nigel Rendell [Centre for Amyloidosis and Acute Phase Proteins, UCL Division of Medicine (Royal Free Campus)] for assistance with mass spectrometry.

\section{References}

Awan, S. J., Siligardi, G., Shoolingin-Jordan, P. M. \& Warren, M. J. (1997). Biochemistry, 36, 9273-9282.

Azim, N., Deery, E., Warren, M. J., Erskine, P., Cooper, J. B., Wood, S. P. \& Akhtar, M. (2013). Acta Cryst. F69, 906-908.

Battersby, A. R., Fookes, C. J. R., Hart, G., Matcham, G. W. J. \& Pandey, P. S. (1983). J. Chem. Soc. Perkin Trans. 1, 1983, 3041-3047.

Bunk, B., Schulz, A., Stammen, S., Münch, R., Warren, M. J., Rohde, M., Jahn, D. \& Biedendieck, R. (2010). Bioeng. Bugs, 1, 85-91.

Chen, V. B., Arendall, W. B., Headd, J. J., Keedy, D. A., Immormino, R. M., Kapral, G. J., Murray, L. W., Richardson, J. S. \& Richardson, D. C. (2010). Acta Cryst. D66, 12-21.

Emsley, P. \& Cowtan, K. (2004). Acta Cryst. D60, 2126-2132.

Evans, P. (2006). Acta Cryst. D62, 72-82.

Gill, R., Kolstoe, S., Mohammed, F., Al d-Bass, A., Mosely, J., Sarwar, M., Cooper, J., Wood, S. \& Shoolingin-Jordan, P. (2009). Biochem. J. 420, 17-25.

Hädener, A., Matzinger, P. K., Battersby, A. R., McSweeney, S., Thompson, A. W., Hammersley, A. P., Harrop, S. J., Cassetta, A.,
Deacon, A., Hunter, W. N., Nieh, Y. P., Raftery, J., Hunter, N. \& Helliwell, J. R. (1999). Acta Cryst. D55, 631-643.

Jordan, P. M. (1991). New Compr. Biochem. 19, 1-66.

Jordan, P. M. \& Warren, M. J. (1987). FEBS Lett. 225, 87-92.

Karplus, P. A. \& Diederichs, K. (2012). Science, 336, 10301033.

Laskowski, R. A., MacArthur, M. W., Moss, D. S. \& Thornton, J. M. (1993). J. Appl. Cryst. 26, 283-291.

Leslie, A. G. W. (2006). Acta Cryst. D62, 48-57.

Louie, G. V. (1993). Curr. Opin. Struct. Biol. 3, 401-408.

Louie, G. V., Brownlie, P. D., Lambert, R., Cooper, J. B., Blundell, T. L., Wood, S. P., Malashkevich, V. N., Hädener, A., Warren, M. J. \& Shoolingin-Jordan, P. M. (1996). Proteins, 25, 48-78.

Louie, G. V., Brownlie, P. D., Lambert, R., Cooper, J. B., Blundell, T. L., Wood, S. P., Warren, M. J., Woodcock, S. C. \& Jordan, P. M. (1992). Nature (London), 359, 33-39.

Lovell, S. C., Davis, I. W., Arendall, W. B., de Bakker, P. I. W., Word, J. M., Prisant, M. G., Richardson, J. S. \& Richardson, D. C. (2003). Proteins, 50, 437-450.

Murshudov, G. N., Skubák, P., Lebedev, A. A., Pannu, N. S., Steiner, R. A., Nicholls, R. A., Winn, M. D., Long, F. \& Vagin, A. A. (2011). Acta Cryst. D67, 355-367.

Powell, H. R., Johnson, O. \& Leslie, A. G. W. (2013). Acta Cryst. D69, 1195-1203.

Read, R. J. (1986). Acta Cryst. A42, 140-149.

Roberts, A., Gill, R., Hussey, R. J., Mikolajek, H., Erskine, P. T., Cooper, J. B., Wood, S. P., Chrystal, E. J. T. \& Shoolingin-Jordan, P. M. (2013). Acta Cryst. D69, 471-485.

Schüttelkopf, A. W. \& van Aalten, D. M. F. (2004). Acta Cryst. D60, 1355-1363.

Scott, A. I., Clemens, K. R., Stolowich, N. J., Santander, P. J., Gonzalez, M. D. \& Roessner, C. A. (1989). FEBS Lett. 242, 319-324.

Scott, A. I., Roessner, C. A., Stolowich, N. J., Karuso, P., Williams, H. J., Grant, S. K., Gonzalez, M. D. \& Hoshino, T. (1988). Biochemistry, 27, 7984-7990.

Shoolingin-Jordan, P. M., Warren, M. J. \& Awan, S. J. (1997). Methods Enzymol. 281, 317-327.

Song, G., Li, Y., Cheng, C., Zhao, Y., Gao, A., Zhang, R., Joachimiak, A., Shaw, N. \& Liu, Z.-J. (2009). FASEB J. 23, 396-404.

Vagin, A. \& Teplyakov, A. (2010). Acta Cryst. D66, 22-25.

Vaguine, A. A., Richelle, J. \& Wodak, S. J. (1999). Acta Cryst. D55, 191-205.

Vary, P. S. (1994). Microbiology, 140, 1001-1013.

Vary, P. S., Biedendieck, R., Fuerch, T., Meinhardt, F., Rohde, M., Deckwer, W.-D. \& Jahn, D. (2007). Appl. Microbiol. Biotech. 76, 957-967.

Warren, M. J. \& Jordan, P. M. (1988). Biochemistry, 27, 90209030.

Warren, M. J. \& Smith, A. G. (2009). Tetrapyrroles: Birth, Life and Death. Austin: Landes Biosciences.

Winn, M. D. et al. (2011). Acta Cryst. D67, 235-242.

Wood, S., Lambert, R. \& Jordan, P. M. (1995). Mol. Med. Today, 1, 232-239. 